\title{
Virtual Reality, Augmented Reality and beyond: Laboratory of Computer Applications for Health Care at University of São Paulo
}

\author{
Fátima L. S. Nunes, Renan V. Aranha, Rafael L. Testa, Matheus A. O. Ribeiro \\ School of Arts, Sciences and Humanities - University of São Paulo \\ Rua Arlindo Béttio, 1000, São Paulo - SP, CEP 03828-000 \\ http://lapis.each.usp.br \\ \{fatima.nunes, renanvinicius, rafael.testa, matheus.alberto.ribeiro\}@usp.br
}

\begin{abstract}
The Laboratory of Computer Applications for Health Care at University of São Paulo develop multidisciplinary research involving Computer Science areas and other knowledge fields such as Physiotherapy, Psychiatry, Radiology, and Cardiology. Most studies use concepts besides Computer Science, by aggregating knowledge to solve real problems. In this paper we present the main projects in Virtual Reality and Augmented Reality areas, highlighting serious games, computer-aided diagnosis and therapy, simulation and training. Besides the social and scientific impact inherent to the type of the developed research, some systems are available to health professionals in order to allow technology transfer and consequent technological and economic impact in daily activities of health tasks.
\end{abstract}

Keywords: health care; simulation and training; serious game; virtual reality; augmented reality; computer-aided diagnosis.

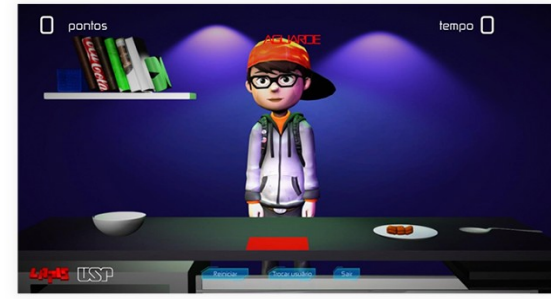

a

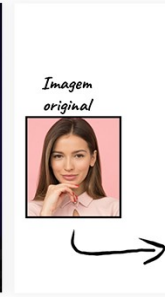

b

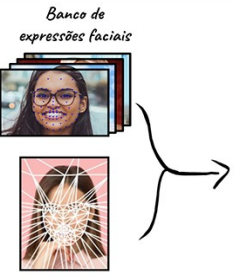

Figure 1. Some projects developed by LApIS members: (a) serious game for virtual rehabilitation; (b) facial emotion synthesis; (c) simulation for

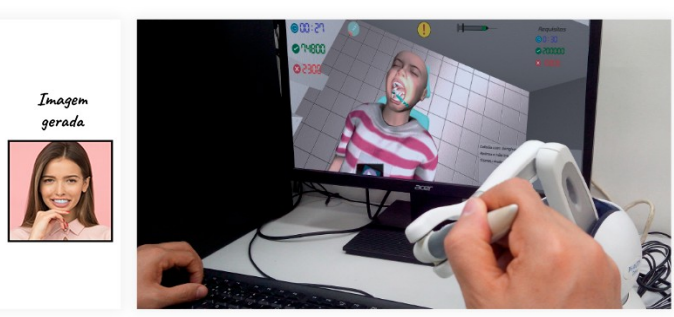

C dental anesthesia.

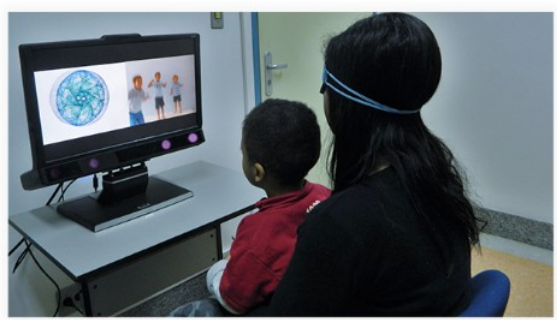

a

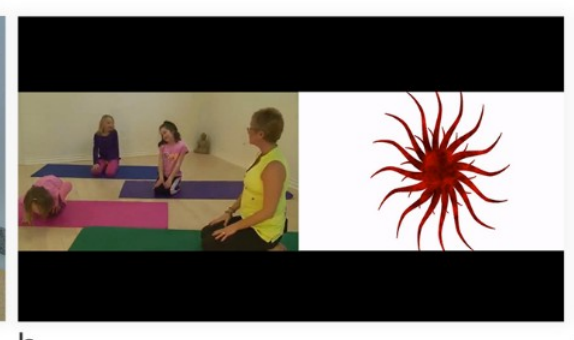

b

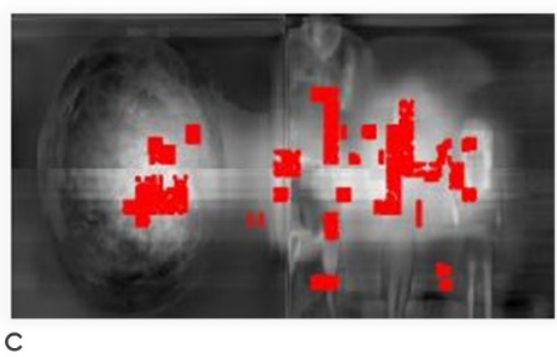

Figure 2. Computer-aided diagnosis in Autism Spectrum Disorder: (a) Patient during eye tracking process; (b) frame of a video used as stimulus; (c) Saliency map obtained from processing of eye tracking signals.

\section{Introduction}

LApIS is an abbreviation in Portuguese for "Laboratory of Computer Applications for Health Care". The laboratory aggregates nine full time advisors from School of Arts, Sciences and Humanities at University of São Paulo (USP), with background in the Computer Science areas, which teach and supervise graduate and undergraduate students. Most of the projects of LApIS are multidisciplinary because their members believe that solution of real problems requires knowledge of several areas. Then, the development of such research requires effective participation of professionals from other areas, who know the application deeply. Thus, besides the advisors above mentioned, LApIS has collaboration with researchers from Brazilian and foreign institutions, with backgrounds not only in Computer Science areas, but also in Psychiatry, Cardiology, Physiotherapy, Odontology, Radiology, among others.

Research developed at LApIS aims to solve some real problems, by answering research questions such as:

- How to develop efficient and engaging systems for virtual rehabilitation?

- How do aid diagnosis and therapy of different anomalies and disorders?

- Using emotions recognition in simulation, games, and computer-aided diagnosis can contribute to the efficiency of these systems? 
- Is it possible to generate intuitive visualization in three-dimensional (3D) environments to decrease the cognitive effort required to understand abstract information?

- Virtual games can present the same efficiency like real games?

In order to answer these and other questions, we put together several areas of Computer Science, such as Graphical Processing, Machine Learning, Computer Theory, Software Engineering, Information Retrieval, and Human-Computer Interaction. Other areas are also studied in order to aggregate useful concepts for the research. Among these, the most recent are studies about characteristics of disorders like Autism Spectrum, Alzheimer, and Mild Cognitive Impairment. Anomalies like cardiomyopathies and breast cancer are also explored in some research. Finally, concepts that involve studies from Psychology and Education, like cognitive skills, affective computing and visual attention have been included in some of the research developed at LApIS.

\section{Main Projects}

The projects developed at LApIS can involve different concepts within Computer Science areas. Most of them use Graphical Processing, considering Virtual Reality (VR) and Augmented Reality (AR), Image Processing and Content-Based Image Retrieval. In this paper the projects more directly related to VR and AR are highlighted (Figure 1). Other projects can be seen at: https://lapis.each.usp.br/.

\subsection{Serious games}

In partnership with physiotherapists, LApIS has developed serious games to aid training and motor rehabilitation processes. In [1], a VR serious game is described for the motor rehabilitation of patients considering the performance of daily activities. Also in this context, Funabashi et al. [2] proposed a serious game whose task is to associate images, involving cognitive and motor challenges. The correspondence between virtual and real games is also investigated [3].

Aiming to provide greater engagement in serious games, some researchers have investigated the automatic adaptation of these applications based on the user's emotional state. In [4], an Affective Computing framework was proposed for the automatic adaptation of serious games aimed to aid motor rehabilitation processes.

In addition to reducing the cost of implementation to the developer, this approach allows the physiotherapist to configure the adaptations to be defined for each patient. Figure 1a illustrates one of the games used in this research.

A $\mathrm{PhD}$ research currently developing aims to not only provide the automatic adaptation of serious games of general purpose, but also to include the analysis of the user's personality in this context [5]. In this sense, a systematic review was developed in order to analyze how the user's personality traits imply the experience of using VR systems [6], as well as a systematic literature review to identify emotion recognition techniques [7].

\subsection{Computer-aided diagnosis and therapy}

Collaboration with Psychiatric Institute at USP and Instituto de Computação (Unicamp) is advancing the state-of-art in computer-aided diagnosis and therapy of Autism Spectrum Disorder (ASD), Alzheimer, as well analysis of Psychotherapy sessions. Initial results obtained by processing anthropometric facial measurements extracted from two-dimensional (2D) images $[8,9]$ are promising.

Using machine learning methods to process eye tracking signals [10] has shown the potential of an approach under development, illustrated by Figure 2 . Some of the psychiatric disorders can limit an individual's ability to recognize facial expressions of emotions. To aid training of this skill, recognition of emotions is explored. LApIS has developed tools for generation of synthetic facial images [11] and caricatures [12] representing emotions to be used in this context (Figure 1b).

Radiology and Cardiology are other two fields of knowledge investigated by some LApIS research. The former is related to aid diagnosis of breast cancer using Computer Theory [13]. Regarding the later, a partnership with Heart Institute at USP allows 3D objects to be reconstructed from Magnetic Resonance Imaging and used to retrieve the most similar objects from a database [14]. Thus, physicians can analyze the retrieved objects to compose their decision about the diagnosis. Figure 3 shows the developed system.
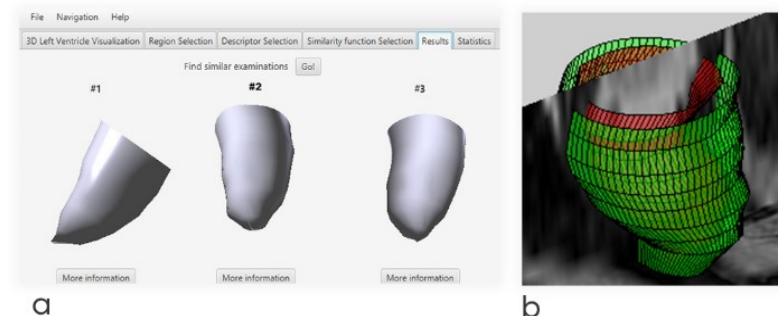

b

Figure 3. Computer-aided diagnosis in Cardiology by using 3D objects retrieval: (a) Ventricle wall reconstruction; (b) 3D environment used for retrieval.

Information Visualization has being explored as a tool to aid diagnosis. Currently, LApIS has explored the visualization of temporal data in 3D space, associating VR concepts to Visual Attention and Machine Learning. Thus, we seek to advance the visualization of temporal data in 3D space, through personalized visualizations based on capturing the users' preferences. While in [15] the use of rule-based learning is discussed in the personalization of visualization of temporal data, in [16] the results of a controlled experiment carried out with health professionals are presented. The proposed approach is extensible to other domains that share data that can be organized into informational clusters. 


\subsection{Simulation and training}

VR simulators for training medical procedures can provide a low cost environment while provide greater safety for students. With this point of view, LApIS has developed VR systems for process simulation and medical training.

In order to reduce the high failure rates in the dental anesthesia procedure, the VR Vida Odonto haptic simulator [17] (Figure 1c) has been developed by LApIS in collaboration with the Faculty of Dentistry of Bauru (USP) and the Interactive Technologies Laboratory of the Polytechnic School (USP). Gamification techniques were incorporated into this simulator to promote engagement and provide student assessment metrics [18], making it a serious game. In another work [19], a method of learning haptic trajectories was added to the game, favoring the learning of trajectories performed by an expert.

In [20], a simulator for the medical procedure of breast palpation with force feedback is presented. This application was built using the ViMeT (Virtual Medical Training) framework, also developed by LApIS [21]. In this simulator, techniques and force feedback parameters are implemented to simulate different features useful to train the palpation exam, such as size and stiffness of nodules, and parameters related to breast composition.

LApIS has also studied methods for simulating physiological processes related to health areas. In [22], partial results of a method for simulating blood flow in arteries are presented. In it, a method joining Smoothed Particle Hydrodynamics and elastic deformations in meshes representing arteries is proposed. The objective is to offer greater realism in the creation of 3D simulators that require a reliable representation of blood flow in the human body.

\section{Impacts and final remarks}

Results of research developed at LApIS have been published in high impact journals and conferences, cited along this paper, which can show the scientific impact of the researchers. Besides this, some awards have been achieved $[8,23,24,25]$, which ratify the quality of the research.

Regarding technological and social contributions, the results of the applications related to diagnosis are currently being tested by the partners in order to include them in the routine of health professionals aiming at aiding ASD, Alzheimer and Cardiology diagnosis. Simulator of dental anesthesia, as well as its gamification, is also being validated aiming at its insertion in the student's routine.

Members of LApIS are always open to exchange experiences with other laboratories. All the projects cited in this paper have opportunities to establish collaboration and include new students.

\section{References}

[1] Aranha, R. V.; Araujo, L. V.; Monteiro, C. B. M.; Da Silva, T. D.; Nunes, F. L. S. (2016) MoVEROffice: Virtual Reality for Upper Limbs Rehabilitation. In: Proc. XVIII Symposium on Virtual and Augmented Reality (SVR). DOI: $10.1109 /$ svr.2016.36

[2] Funabashi, A. M. M.; Aranha, R. V.; Silva, T. D.; Monteiro, C. B. M.; Silva, W. S.; Nunes, F. L. S. (2018) A serious game for virtual rehabilitation: evaluation with patients and physiotherapists. SBC Journal on Interactive Systems 9(2): 14-25.

[3] Massetti, T.; Fávero, F. M.; Menezes, L. D. C.; Alvarez, M. P. B.; Crocetta, T. B.; Guarnieri, R.; Nunes, F. L. S.; Monteiro, C. B. M.; Silva, T. D. (2018) Achievement of Virtual and Real Objects Using a Short-Term Motor Learning Protocol in People with Duchenne Muscular Dystrophy: A Crossover Randomized Controlled Trial. Games for Health Journal 7(2): 107-115. DOI: 10.1089/g4h.2016.0088

[4] Aranha, R. V.; Silva, L. S.; Chaim, M. L.; Nunes, F. L. S. (2017) Using Affective Computing to Automatically Adapt Serious Games for Rehabilitation. In: Proc. 30th International Symposium on Computer-Based Medical Systems (CBMS). DOI:10.1109/cbms.2017.89

[5] Aranha, R. V; Nunes, F. L. S. (2019) Um Framework para Adaptação Automática de Jogos Sérios Considerando as Emoções e os Traços de Personalidade do Jogador. Anais Estendidos Do Simpósio Brasileiro de Fatores Humanos Em Sistemas Computacionais (IHC) DOI: $10.5753 /$ ihc. 2019.8422

[6] Aranha, R. V.; Nakamura, R.; Tori, R.; Nunes, F. L. S. (2018) Personality Traits Impacts in Virtual Reality's User Experience. In: Proc. 20th Symposium on Virtual and Augmented Reality (SVR). DOI: 10.1109/svr.2018.00019

[7] Aranha, R. V.; Correa, C. G.; Nunes, F. L. S. (2019) Adapting software with Affective Computing: a systematic review. IEEE Transactions on Affective Computing 1-1. DOI: $10.1109 /$ taffc. 2019.2902379

[8] Machado-Lima, A.; Silva, A. F.; Nunes, F. L. S.; Brentani, H.; Pedrini, H.; Revers, M. C.; Testa, R. L.; Nobayashi, T.; Pinheiro, T.D. (2017) Classificação multiclasse de transtornos psiquiátricos baseada em medidas antropométricas faciais. In: MCTIC. (Org.). Tecnologias para a economia do conhecimento: edição 2017 do Prêmio Mercosul de Ciência e Tecnologia. 1ed.Brasília (DF): MCTIC, 2019, v. 1, p. 208-233.

[9] Cornejo, J. Y. R.; Pedrini, H.; Machado-Lima, A.; Nunes, F. L. S. (2017) Down syndrome detection based on facial features using a geometric descriptor. Journal of Medical Imaging 4(04): 1. DOI:10.1117/1.jmi.4.4.044008

[10] Pereira, M. L. G. de F.; Camargo, M. von Z. de A.; Bellan, A. F. R.; Tahira, A. C.; dos Santos, B.; dos Santos, J.; Machado-Lima, A; Nunes, F. L. S.; Forlenza, O. V. (2020) Visual Search Efficiency in Mild Cognitive Impairment and Alzheimer's Disease: An Eye Movement Study. Journal of Alzheimer's Disease 75(1): 261-275. DOI: $10.3233 /$ jad-190690

[11] Testa, R. L.; Machado-Lima, A.; Nunes, F. L. S. (2018) Factors Influencing the Perception of Realism in Synthetic Facial Expressions. In: Proc. 31st SIBGRAPI Conference on Graphics, Patterns and Images (SIBGRAPI). DOI: 10.1109/sibgrapi.2018.00045

[12] Testa, R. L.; Muniz, A. H. N.; Carpio, L. U. S.; Dias, R. da S.; Rocca, C. C. de A.; Machado-Lima, A; Nunes, F. L. S. (2015) Generating Facial Emotions for Diagnosis and Training. In: Proc IEEE 28th International Symposium on Computer-Based Medical Systems. DOI: $\underline{10.1109 / \text { cbms.2015.59 }}$ 
[13] Dias Pedro, R. W.; Machado-Lima, A.; Nunes, F. L. S. (2020) Towards an approach using grammars for automatic classification of masses in mammograms. Computational Intelligence. DOI: $\underline{10.1111 / \text { coin.12320 }}$

[14] Bergamasco, L. C. C.; Rochitte, C. E.; Nunes, F. L. S. (2018) 3D medical objects processing and retrieval using spherical harmonics. In: Proc. 33rd Annual ACM Symposium on Applied Computing - SAC'18. DOI: 10.1145/3167132.3167168

[15] Silva, L. S.; Nakamura, R.; Nunes, F. L. S. (2020) Machine Learning in Information Visualization: Using Rule-based Learning Algorithms to Personalize Interfaces. In: Proc. 2020 International Conference on Systems, Signals and Image Processing (IWSSIP). DOI: 10.1109/iwssip48289.2020.9145098

[16] Silva, L. S.; Aranha, R. V.; Ribeiro, M. A. O; Nakamura, R.; Nunes, F. L. S. (2020) Exploring Visual Attention and Machine Learning in 3D visualization of medical temporal data. In: Proc. IEEE 33rd International Symposium on Computer-Based Medical Systems (CBMS). DOI: 10.1109/CBMS49503.2020.00035

[17] Corrêa, C. G.; Machado, M. A. de A. M.; Ranzini, E.; Tori, R.; Nunes, F. L. S. (2017) Virtual Reality simulator for dental anesthesia training in the inferior alveolar nerve block. Journal of Applied Oral Science 25(4): 357-366. DOI: 10.1590/1678-7757-2016-0386

[18] Ribeiro, M. A. O.; Correa, C. G.; Nunes, F. L. S. (2017) Gamification as a Learning Strategy in a Simulation of Dental Anesthesia. In: Proc. 19th Symposium on Virtual and Augmented Reality (SVR). DOI: 10.1109/svr.2017.42

[19] Ribeiro, M. A. O., Correa, C. G.; Nunes, F. L. S. (2018). Visual and Haptic Trajectories Applied to Dental Anesthesia Training: Conceptualization, Implementation and Preliminary Evaluation. In: Proc. 20th Symposium on Virtual and Augmented Reality (SVR). DOI: $\underline{10.1109 / \text { svr.2018.00039 }}$
[20] Ribeiro, M. L..; Nunes, F. L. S.; Elias, S. (2016) Towards Determining Force Feedback Parameters for Realistic Representation of Nodules in a Breast Palpation Simulator. In: Proc. IEEE 29th International Symposium on Computer-Based Medical Systems (CBMS). DOI: 10.1109/cbms.2016.74

[21] Oliveira, A. C. M. T. G.; Nunes, F. L. S. (2009) Building a Open Source Framework for Virtual Medical Training. Journal of Digital Imaging 23(6): 706-720. DOI: $10.1007 /$ s10278-009-9243-3

[22] Carvalho Junior, A. S. V.; Biscaro, H. H. (2019) Blood Flow SPH Simulation with Elastic Deformation of Blood Vessels. In: Proc. IEEE 19th International Conference on Bioinformatics and Bioengineering (BIBE). DOI: 10.1109/bibe.2019.00102

[23] Menção honrosa no VIII Workshop de Pós-Graduação de Engenharia de Computação - Escola Politécnica USP. Artigo "Adapting software with Affective Computing: a systematic review", Programa de Pós-Graduação em Engenharia Elétrica - Escola Politécnica Universidade de São Paulo.

[24] Prêmio de primeiro lugar no "Undergraduate Student Workshop" no Symposium on Virtual and Augmented Reality 2018 - Artigo: Uso de trajetórias visuais e hápticas para auxílio no treinamento de anestesia odontológica, Sociedade Brasileira de Computação.

[25] Prêmio de primeiro lugar na categoria "Application" no Symposium on Virtual and Augmented Reality 2017 Artigo "AGaR: a VR serious game to support the recovery of post-stroke patients", Sociedade Brasileira de Computação. 